\title{
Interplay between magnetic properties and Fermi surface nesting in iron pnictides
}

\author{
A. N. Yaresko, G.-Q. Liu, V. N. Antonov, and O. K. Andersen \\ Max-Planck-Institut für Festkörperforschung, Heisenbergstraße 1, D-70569 Stuttgart, Germany
}

(Dated: November 2, 2018)

\begin{abstract}
The wave-vector $(\mathbf{q})$ and doping $(x, y)$ dependences of the magnetic energy, iron moment, and effective exchange interactions in $\mathrm{LaFeAsO}_{1-x} \mathrm{~F}_{x}$ and $\mathrm{Ba}_{1-2 y} \mathrm{~K}_{2 y} \mathrm{Fe}_{2} \mathrm{As}_{2}$ are studied by self-consistent LSDA calculations for co-planar spin spirals. For the undoped compounds $(x=0, y=0)$, the minimum of the calculated total energy, $E(\mathbf{q})$, is for $\mathbf{q}$ corresponding to stripe antiferromagnetic order. Already at low levels of electron doping $(x)$, this minimum becomes flat in $\mathrm{LaFeAsO}_{1-x} \mathrm{~F}_{x}$ and for $x \gtrsim 5 \%$, it shifts to an incommensurate q. In $\mathrm{Ba}_{1-2 y} \mathrm{~K}_{2 y} \mathrm{Fe}_{2} \mathrm{As}_{2}$, stripe order remains stable for hole doping up to $y=0.3$. These results are explained in terms of the band structure. The magnetic interactions cannot be accurately described by a simple classical Heisenberg model and the effective exchange interactions fitted to $E(\mathbf{q})$ depend strongly on doping. The doping dependence of the $E(\mathbf{q})$ curves is compared with that of the noninteracting magnetic susceptibility for which similar trends are found.

PACS numbers: 74.70.-b, 71.20.-b, 75.25.+z, 75.30.Fv
\end{abstract}

\section{INTRODUCTION}

The discovery of superconductivity with $T_{c}=27 \mathrm{~K}$ in Fdoped $\mathrm{LaFeAsO}_{1-x} \mathrm{~F}_{x}$ by Hosono and co-workers ${ }^{1}$ a year ago initiated an avalanche of experimental and theoretical investigations of layered iron pnictides and recently also chalcogenides. Soon, the superconducting transition temperature was raised above $50 \mathrm{~K}$ by substituting La by smaller rare-earth ions $\stackrel{2.3}{=}$ The interest in layered iron pnictides increased even more when superconductivity below $T_{c}=38 \mathrm{~K}$ was reported in oxygen-free potassiumdoped $\mathrm{Ba}_{1-2 y} \mathrm{~K}_{2 y} \mathrm{Fe}_{2} \mathrm{As}_{2}, \stackrel{4}{=}$ for which good quality single crystals could be synthesized ${ }^{5}$

Both families of iron pnictides have a quasi-twodimensional (2D) tetragonal crystal structure, in which FeAs layers are separated by either $\mathrm{LaO}$ or $\mathrm{Ba}$ layers. The Fe ions form a square lattice sandwiched between two As sheets shifted so that each Fe is surrounded by a slightly squeezed As tetrahedron. At about $150 \mathrm{~K}$, both stoichiometric parent compounds undergo a structural transition at which the symmetry of the lattice lowers

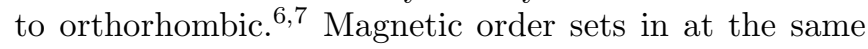
temperature as the structural transition in $\mathrm{BaFe}_{2} \mathrm{As}_{2}$, but at a $20 \mathrm{~K}$ lower temperature in LaFeAsO. In both cases, the order is striped: ferromagnetic (FM) along the shorter axis of the square Fe sublattice and antiferromagnetic (AFM) along the longer axis and between the $\mathrm{Fe}$ layers. 6.8.9 The Fe moments are 0.4-0.9 $\mu_{\mathrm{B}}$ in $\mathrm{BaFe}_{2} \mathrm{As}_{2}$ and $0.3-0.4 \mu_{\mathrm{B}}$ in $\mathrm{LaFeAsO} .8 .10$

Electron doping of the FeAs layers in $\mathrm{LaFeAsO}_{1-x} \mathrm{~F}_{x}$ suppresses the structural and magnetic transitions in favor of superconductivity already at $x=0.03 \stackrel{11}{1}$ Also hole doping in $\mathrm{Ba}_{1-2 y} \mathrm{~K}_{2 y} \mathrm{Fe}_{2} \mathrm{As}_{2}$ suppresses the structural and magnetic transition, $\underline{\underline{4}}$ but this requires hole doping in excess of $y \approx 0.15 \stackrel{12}{\underline{n}}$ Since the superconducting transition occurs already for $y \approx 0.10$, the superconducting and striped AFM phases seem to coexist over a fairly wide range of hole doping .8 .12 Although it is not clear whether the superconductivity is mediated by AFM fluc- tuations or it competes with magnetism, understanding merely the magnetic interactions is currently of utmost importance.

A large number of electronic band-structure calculations using the local spin-density approximation (LSDA) or generalized-gradient approximation now exist for both $\mathrm{LaFeAsO}$ and $\mathrm{BaFe}_{2} \mathrm{As}_{2}, \frac{11,13,14,15,16,17,18,19,20,21}{1}$ The results obtained for $\mathrm{LaFeAsO}$ were reviewed and analyzed in Ref. 22. Although the Fe magnetic moment and the stabilization energies of different magnetic solutions depend strongly on the computational method and the exchange-correlation functional, as well as on whether the experimental or calculated structure is used, all calculations predict that stripe AFM order is the magnetic ground state in both parent compounds. However, the calculated sublattice magnetizations are significantly larger than the ones deduced from the neutron diffraction, $\mu \mathrm{SR}$, and Mössbauer experiments,$\underline{6,8,9,10}$

For both parent compounds, many authors (see, e.g., Refs. 11, 16, 20) have noticed a strong Fermi surface (FS) nesting for the $\mathbf{q}$ vector which corresponds to stripe AFM order between the Fe $d_{x z / y z}$-like hole sheet and one of the two electron sheets. This nesting causes peaks in both the imaginary and real parts of the non-interacting spin susceptibility, $\chi_{0}(\mathbf{q})$, at the stripe $\mathbf{q}$. Although electron doping of LaFeAsO suppresses the peak and shifts it to an incommensurate wave vector, $\stackrel{11}{=}$ it is widely believed that stripe AFM order remains the LSDA ground state of $\mathrm{LaFeAsO}_{1-x} \mathrm{~F}_{x}$. That merely $3 \%$ electron doping suffices to destroy the static stripe order, has been related to filling of the three-dimensional 3D Fe $d_{3 z^{2}-1}$-like band. ${ }^{22}$ However, calculations which use the experimental structure -such as those presented below- place the top of the $d_{3 z^{2}-1}$-like band several hundred meV below the Fermi level.

In this paper, we shall present LSDA calculations of moments and energies of spin spirals in $\mathrm{LaFeAsO}_{1-x} \mathrm{~F}_{x}$ and $\mathrm{Ba}_{1-2 y} \mathrm{~K}_{2 y} \mathrm{Fe}_{2} \mathrm{As}_{2}$ as functions of wave vector, $\mathbf{q}$, and doping, $x$ or $y$. We find that upon increasing doping, 
TABLE I: Lattice and LMTO spheres

\begin{tabular}{ccc}
\hline \hline Sphere & Wycoff position & Radius $(\AA)$ \\
\hline \multicolumn{2}{c}{ LaFeAsO $(P 4 / n m m), a=b=4.04 \AA, c=8.74 \AA$} \\
$\mathrm{Fe}$ & $2 b(0.25,0.75,0.50)$ & 1.41 \\
$\mathrm{As}$ & $2 c(0.25,0.25,0.651)$ & 1.48 \\
$\mathrm{La}$ & $2 c(0.25,0.25,0.142)$ & 1.71 \\
$\mathrm{O}$ & $2 a(0.25,0.75,0)$ & 1.13 \\
$\mathrm{E}_{1}$ & $2 c(0.25,0.25,0.407)$ & 1.08 \\
$\mathrm{E}_{2}$ & $4 f(0.25,0.75,0.266)$ & 1.04 \\
$\mathrm{E}_{3}$ & $2 c(0.25,0.25,-.115)$ & 0.97 \\
\hline \multicolumn{2}{c}{$\mathrm{BaFe}_{2} \mathrm{As}_{2}(I 4 / m m m), a=b$} & $3.96 \AA, c=13.02 \AA$ \\
$\mathrm{Fe}$ & $4 d(0,0.5,0.25)$ & 1.38 \\
$\mathrm{As}$ & $4 e(0,0,0.355)$ & 1.46 \\
$\mathrm{Ba}$ & $2 a(0,0,0)$ & 2.01 \\
$\mathrm{E}_{1}$ & $4 e(0,0,0.195)$ & 0.99 \\
$\mathrm{E}_{2}$ & $2 b(0,0,0.50)$ & 0.78 \\
$\mathrm{E}_{3}$ & $8 g(0.5,0,-.105)$ & 0.84 \\
\hline \hline
\end{tabular}

stripe AFM order becomes unstable in favor of an incommensurate spin-density wave (SDW).

Before getting to the spin spirals, we shall explain our computational method and compare with previous results -and also present results involving spin-orbit coupling - for the paramagnetic band structures and the commensurate stripe and checkerboard SDWs for the parent compounds.

\section{COMPUTATIONAL METHOD}

Our LSDA scalar-relativistic calculations for co-planar spin spirals in $\mathrm{LaFeAsO}_{1-x} \mathrm{~F}_{x}$ with $x=0,0.1,0.2$, and 0.3 and $\mathrm{Ba}_{1-2 y} \mathrm{~K}_{2 y} \mathrm{Fe}_{2} \mathrm{As}_{2}$ with $y=0,0.1,0.2$, and 0.3 were carried out using the linear muffin-tin orbital (LMTO) method in the atomic-sphere approximation and including the combined correction term. 23 For the exchange-correlation potential, we used the Perdew-Wang parametrization. ${ }^{24}$ Charge- and spin-selfconsistent calculations were carried out for all doping levels using the virtual-crystal approximation with fractional atomic number of $\mathrm{O}$ or $\mathrm{Ba}$. All our calculations were for the experimental room-temperature crystal structures of the undoped compounds, 1,6 This is important. Whereas the primitive cell of LaFeAsO holds two formula units and is tetragonal $(P 4 / \mathrm{nmm})$, that of $\mathrm{BaFe}_{2} \mathrm{As}_{2}$ holds one unit and is body-centered tetragonal (bct) $(I 4 / \mathrm{mmm})$. The lattice constants as well as the positions and radii of the space-filling atomic and empty (E) LMTO spheres are given in Table I]

For the Fe $d$ orbitals, such as $d_{x y}$, we use the $x$ and $y$ axes (not to be confused with the levels of electron and hole doping) which span the quadratic Fe sublattice and are therefore turned $45^{\circ}$ with respect to the tetragonal $a$ and $b$ axes. The lobes of the Fe $d_{x y}$ orbital thus point toward the projections of the As sublattice onto the Fe plane, while $d_{x^{2}-y^{2}}$ points toward the nearest Fe neighbors. Or in other words: the Fe-Fe $d d \pi$ interaction

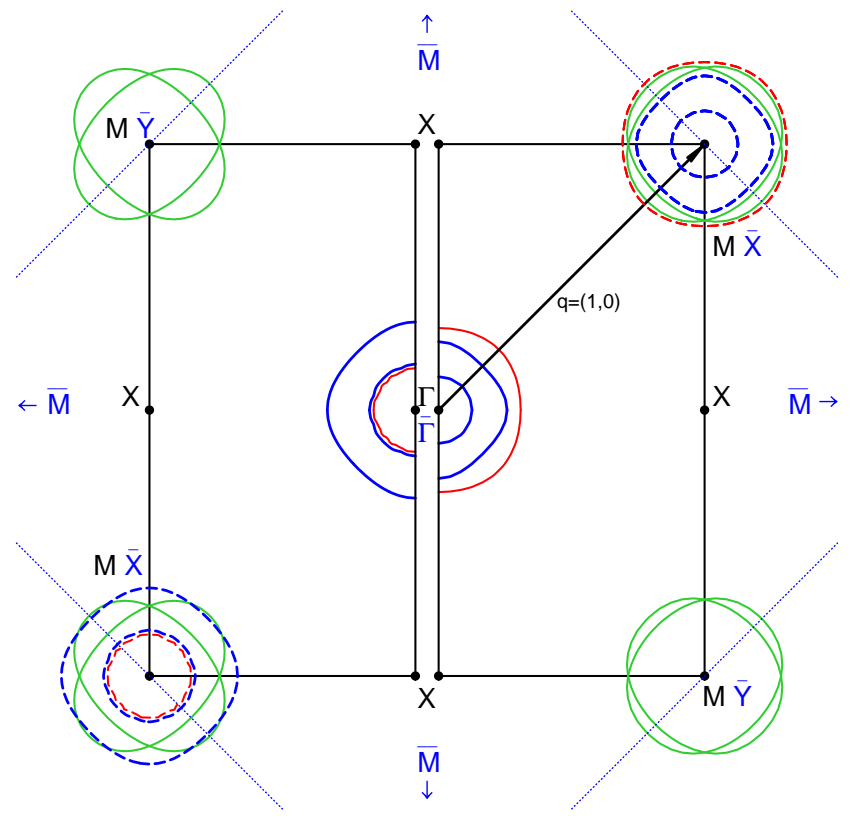

FIG. 1: (Color online) FS cross sections $\left(k_{z}=0\right)$ for LaFeAsO in the tetragonal Brillouin zone (BZ). The $a$ direction is horizontal, the $b$ direction is vertical, and the coordinates of the (black) $\Gamma, \mathrm{X}$, and $\mathrm{M}$ points are as given in Fig. 2. The FS on the left- and right-hand sides were calculated, respectively, with and without a $150 \mathrm{meV}$ downwards adjustment of $\epsilon_{\nu x y}$ in the LMTO method. The $\Gamma$-centered $d_{x y}$-like hole cylinder is shown in thin (red) line and the concentric $d_{y z, z x}$-like hole cylinders are shown in thick (blue) lines. The two Mcentered, electron cylinders shown in thin (green) lines have super-ellipsoidal cross sections with main axes directed toward $\Gamma$. The fact that a primitive translation of the square Fe sublattice followed by mirroring in the Fe plane generates an Abelian subgroup of the space group allows one to fold the band structure out to a large one-formula-unit BZ. This moves the $d_{x z / y z}$-like hole cylinders to the nearest-neighbor $\Gamma$ points, which are the (blue) $\overline{\mathrm{M}}$ corners of the large BZ and have the coordinates $(1,1) \pi \sqrt{2} / a$ in the $(x, y)$-system. This also separates the electron cylinders onto different $\mathrm{M}$ points, now called $\overline{\mathrm{X}}(1,0)$ and $\overline{\mathrm{Y}}(0,1)$ (blue), such that the super-ellipses have their short axis pointing toward the $\bar{\Gamma}$-centered $d_{x y}$-like hole cylinders and the long axis pointing toward the $\overline{\mathrm{M}}$-centered $d_{x z / y z}$-like hole cylinders. Introducing a commensurate SDW with $\mathbf{q}=(1,0)$ in the $(x, y)$ system, i.e., FM-ordered stripes in the $y$-direction and AFM order in the $x$-direction, will shift the hole cylinders by q. This is shown by dashed lines around the bottom-left and top-right corners, $\mathrm{M}=\overline{\mathrm{X}}$. The selection rules following from the glide mirror allow coupling only between the $d_{x y}$ holes and the electron sheet with the short axis along $\mathbf{q}$, and between the $d_{x z / y z}$ holes and the electron sheet with the long axis along q. Introducing a SDW with $\mathbf{q}=(1,1)$, i.e., checkerboard AFM, folds the large BZ back into the small tetragonal one. Here the nesting is between the $d_{x y}$-like and one of the $d_{x z / y z}$-like hole sheets and between two different electron sheets. Not included in our spin-spiral calculations is the spin-orbit coupling $(\xi \sim 60 \mathrm{meV})$, which invalidates the glide mirror and violates the selection rules. 

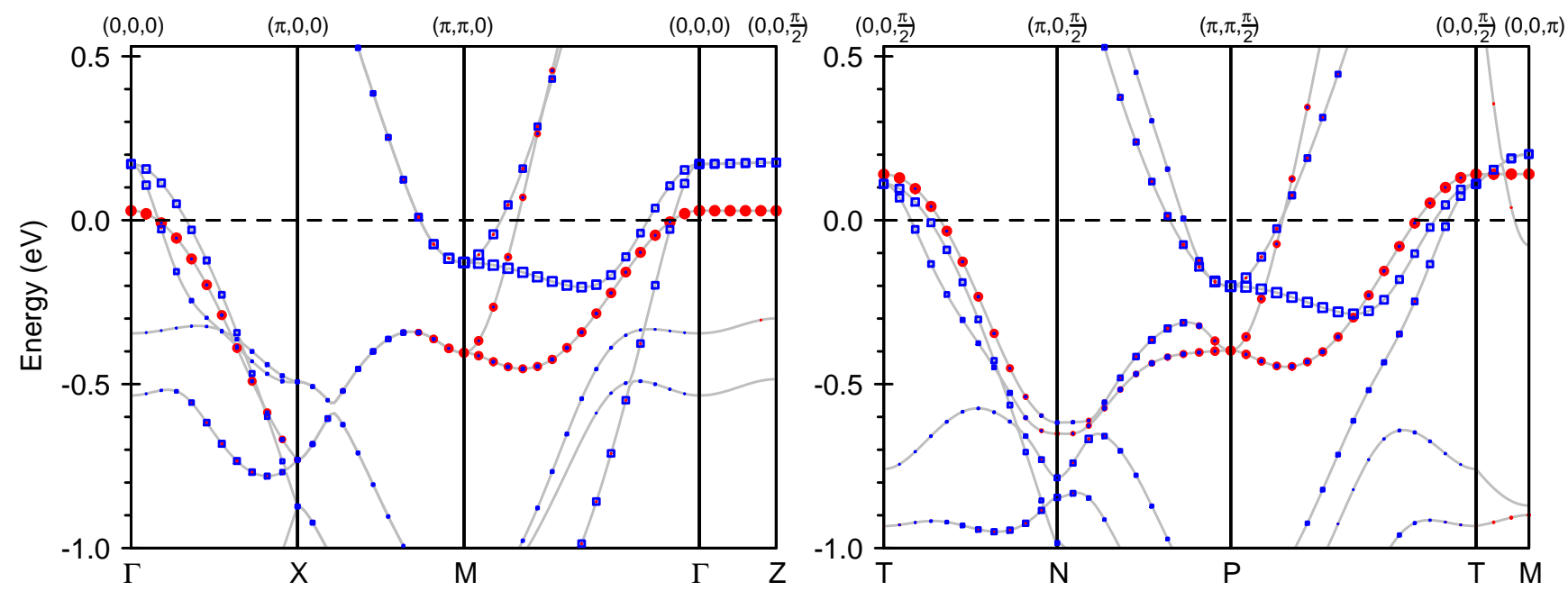

FIG. 2: (Color online) Band structures of undoped LaFeAsO (left panel) and $\mathrm{BaFe}_{2} \mathrm{As}_{2}$ (right panel) obtained from non-spinpolarized calculations for the experimental structure. The size of (red) circles and (blue) squares is proportional to, respectively, the Fe $d_{x y}$ and $d_{y z, z x}$ partial-wave character. The coordinates shown at the bottom are in the tetragonal $(a, b, c)$-system where the cell contains two Fe atoms, i.e., in units of $(1 / a, 1 / b=1 / a, 1 / c)$. The Fermi level is the zero of energy.

involves the $d_{x y}$ orbitals and the Fe-Fe $d d \sigma$ interaction involves the $d_{x^{2}-y^{2}}$ orbitals. This convention is the same as the one used for the $\mathrm{Cu}$ orbitals in the high-temperature superconducting cuprates. The distance, $a / \sqrt{2}$, between Fe nearest neighbors is $2.85 \AA$ in $\mathrm{LaFeAsO}$ and $2.80 \AA$ in $\mathrm{BaFe}_{2} \mathrm{As}_{2}$.

Our band structures and Fermi surfaces for undoped paramagnetic $\mathrm{LaFeAsO}$ and $\mathrm{BaFe}_{2} \mathrm{As}_{2}$ obtained from spin-restricted LDA LMTO calculations agree well with those obtained with the same method and presented in Ref. 21.

However, for LaFeAsO the LMTO Fermi surface differs from that obtained with the full-potential linear augmented plane-wave (LAPW) method: $\stackrel{22}{=}$ Whereas LMTO finds the two innermost $\Gamma$-centered hole cylinders to be $d_{x z, y z}$ like and the outermost $d_{x y}$ like, LAPW finds the opposite order. Since LAPW is computationally more cumbersome and accurate than LMTO, we used LMTO but applied an external crystal field which shifted the energy $\left(\epsilon_{\nu x y}\right)$ of the Fe $d_{x y}$ partial wave downwards by 150 $\mathrm{meV}$. This adjustment brought the LMTO and LAPW band structures into almost complete agreement. The adjusted and unadjusted Fermi surfaces are shown respectively on the left and right-hand sides of Fig. 1, In the following, all results presented for $\mathrm{LaFeAsO}_{1-x} \mathrm{~F}_{x}-$ such as the paramagnetic bands on the left-hand side of Fig. 2 and the spin-spiral moments and energies in Fig. 4are those obtained with LMTO and the $d_{x y}$-energy downshifted, unless otherwise stated.

For $\mathrm{BaFe}_{2} \mathrm{As}_{2}$, the LMTO LDA band structure shown on the right-hand side of Fig. 2 agrees very well with the LAPW one. For that reason, our LMTO calculations for $\mathrm{Ba}_{1-2 y} \mathrm{~K}_{2 y} \mathrm{Fe}_{2} \mathrm{As}_{2}$ were all performed without any adjustment.
Finally, we calculated effects of spin-orbit coupling (Fermi surface splittings and magnetocrystalline energies) using the spin-polarized relativistic LMTO $\operatorname{method}^{25}$ and the experimental structure.

\section{PARAMAGNETIC ENERGY BANDS}

The paramagnetic scalar-relativistic bands for undoped LaFeAsO are shown near the Fermi level at the left-hand side of Fig. 2. We see that at $\Gamma$, the top of the $d_{x z / y z}$ band (blue squares) is $\sim 180 \mathrm{meV}$ while that of the $d_{x y}$ band (red spheres) is merely $\sim 30 \mathrm{meV}$ above $\varepsilon_{F}$. Without the $150 \mathrm{meV}$ shift, these levels would have been nearly degenerate, and since the mass of the $d_{x y}$ band is higher that those of the two $d_{x z / y z}$ bands, the cylindrical $d_{x y}$ sheet would have been the widest, as shown in the right-hand side of Fig. 1. Although spin-orbit coupling cannot be included in spin-spiral calculations, we mention that it splits the degenerate top of the $d_{x z / y z}$-like band by $50 \mathrm{meV}$, a value consistent with $\xi_{\mathrm{Fe} 3 d} \approx 60 \mathrm{meV}$ and a $20 \%$ reduction due to by-mixing of As $p$ character.

With an even number of electrons $\left(\mathrm{Fe} d^{6}\right)$, the sum of the volumes of the three hole sheets equals that of the electron sheets. Of those, there are two equivalent cylinders centered at $\mathrm{M}$ and turned $90^{\circ}$ with respect to each other (thin green). Their cross sections are superellipsoidal with main axes pointing toward $\Gamma$. The superellipsoidal cross section arises because these sheets result from a $d_{x z}$ or $d_{y z}$ band hybridizing with a lower-lying $d_{x y}$-like band ${ }^{26}$ The main character is $d_{x z}$ or $d_{y z}$ near the short axis and $d_{x y}$ near the long axis. These electron cylinders have more As $p$ character and more $k_{z}$-dispersion than the hole cylinders, and are therefore more warped. 
The fact that a primitive translation of the square Fe sublattice followed by mirroring in the Fe plane generates an Abelian subgroup of the $P 4 / \mathrm{nmm}$ space group allows one to fold the band structure out to the large Brillouin zone (BZ) well known from the cuprates ${ }^{26,27}$ This folding out has the advantage of separating the $d_{x y}$ cylinders from the $d_{x z / y z}$ ones by placing them at respectively $\bar{\Gamma}$ $(0,0)$ and $\bar{M}(1,1)$ in the $(x, y)$ system. It also separates the two electron pockets from each other by placing them at, respectively, $\overline{\mathrm{X}}(1,0)$ and $\overline{\mathrm{Y}}(0,1)$ with the long axis pointing toward $\overline{\mathrm{M}}$.

Note that in order to distinguish the tetragonal $(a, b, c)$-directed coordinates in reciprocal space from the quadratic $(x, y, z \| c)$-directed ones, we use the units $(1 / a, 1 / a, 1 / c)$ in the former case and $(\sqrt{2} \pi / a, \sqrt{2} \pi / a, \pi / c)$ in the latter case. Hence, tetragonal (quadratic) reciprocal-space coordinates are recognized by the presence (absence) of the factor $\pi$.

Spin-orbit coupling does not commute with the abovementioned glide mirror and will therefore split the crossing between the $d_{x y}$ and one of the $d_{x z / y z}$ hole bands by about $50 \mathrm{meV}$, as well as the crossings between two electron bands as has been observed in LaFePO 28

At the right-hand side of Fig. 2, we show the paramagnetic bands near the Fermi level for undoped $\mathrm{BaFe}_{2} \mathrm{As}_{2}$. Since the $\mathrm{As}_{\mathrm{s}} \mathrm{Fe}_{2}$-As layers are separated by a thin $\mathrm{Ba}$ layer, rather than by a thick $\mathrm{La}-\mathrm{O}_{2}$-La layer, and the AS atoms along the $\mathrm{z}$ axis are stacked on top of each other the band structure of $\mathrm{BaFe}_{2} \mathrm{As}_{2}$ disperses more in the $z$ direction than that of LaFeAsO. But apart from that, the band structures are very similar. For ease of comparison with the LaFeAsO band structure at the left-hand side of the figure, we have chosen the same route in $\left(k_{x}, k_{y}\right)$ space, but have taken $k_{z}=\pi /(2 c)$-except in the very last panel- because this choice makes the amplitude of the Bloch waves vanish in the Ba-plane and thus minimizes the effects of $k_{z}$ dispersion. Along $\left(0,0, k_{z}\right)$, the $d_{x y}$-like band is $140 \mathrm{meV}$ above $\varepsilon_{F}$ and does not disperse with $k_{z}$, whereas the doubly degenerate $d_{x z / y z}$-like band disperses from 40 to $200 \mathrm{meV}$ above $\varepsilon_{F}$ with $k_{z}$ increasing from 0 to $\pi / c$, and thus goes from below to above the top of the $d_{x y}$ band. The hole sheets thus remain cylinders although the $d_{x z / y z}$-like sheets are significantly warped. Near $(0,0, \pi / c)$, we see a band with strong $k_{z^{-}}$ dispersion dip below $\varepsilon_{F}$. This band is As $p_{z}$ like and cannot hybridize with the $d_{x y}$-like band, but only with the $d_{x z / y z}$-like band, but not along $\left(0,0, k_{z}\right)$. This hybridization gives rise to an intricate shape of the $d_{x z / u z}$ bands near $\varepsilon_{F}$ for $k_{z} \neq \pi /(2 c)$ and is discussed in Ref. 26 . A further difference with the LaFeAsO bands is that the electron cylinders around $\left(\pi, \pi, k_{z}\right)$ are not degenerate along the $a$ and $b$ directions and that the $k_{z}$ dispersion of the $d_{x y}$-like component is as large as $150 \mathrm{meV}$ due to by-mixing of As $p_{z}$ character.

The main effect of electron doping on the band structure is to move the Fermi level up or -equivalently- to move the bands down with respect to the Fermi level. This is clearly seen in Fig. 3 where we show dependence

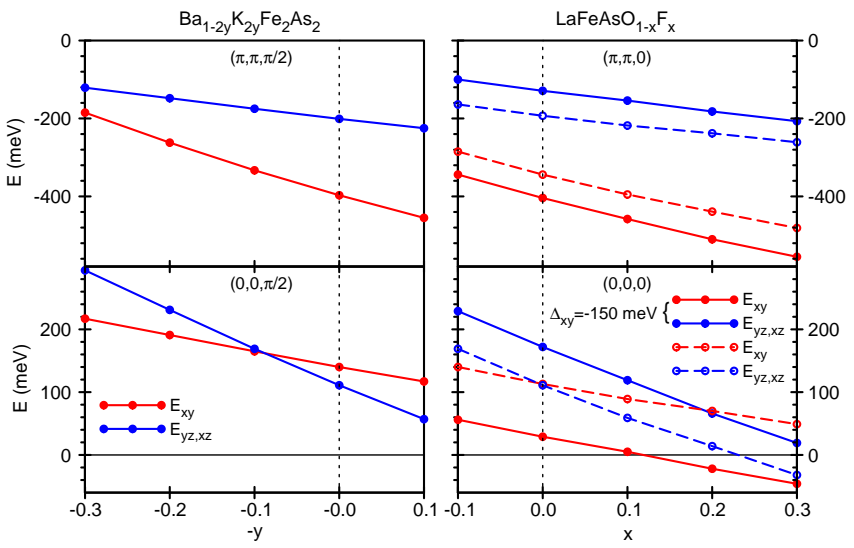

FIG. 3: (Color online) Band positions as functions of doping in the range $-30 \% \leq x=-y \leq 30 \%$ for $\mathrm{Ba}_{1-2 y} \mathrm{~K}_{2 y} \mathrm{Fe}_{2} \mathrm{As}_{2}$ (left) and $\mathrm{LaFeAsO}_{1-x} \mathrm{~F}_{x}$ (right).

on doping - ranging from $-30 \%$ in $\mathrm{Ba}_{1-2 y} \mathrm{~K}_{2 y} \mathrm{Fe}_{2} \mathrm{As}_{2}$ to $+30 \%$ in $\mathrm{LaFeAsO}_{1-x} \mathrm{~F}_{x}$ - of the top of the $d_{x y}$-like (red points) and $d_{x z / y z}$-like (blue squares) hole bands at $(0$, $0, \pi / 2$, or 0$)$ and the bottoms of the $d_{x y^{-}}$and $d_{x z^{-}}$or $d_{y z}$-like electron bands at $(\pi, \pi, \pi / 2$, or 0$)$. We note, first of all, that the dominating rigid shift of these levels is roughly continuous when passing from hole doping in $\mathrm{Ba}_{1-2 y} \mathrm{~K}_{2 y} \mathrm{Fe}_{2} \mathrm{As}_{2}$ to electron doping in $\mathrm{LaFeAsO}_{1-x} \mathrm{~F}_{x}$ (and even more so had we not corrected $\epsilon_{\nu x y}$ for the latter). Second, we note that $11 \%$ electron doping fills the $d_{x y}$ band, and $33 \%$ fills the $d_{x z / y z}$ band, and that no other Fe $d$-like band gets filled or emptied in the $\pm 30 \%$ doping range. Within this range, the bands move by about $300 \mathrm{meV}$, corresponding to an average density of states of order 2 electrons $/ \mathrm{Fe} / \mathrm{eV}$. The deviation from rigid-band behavior, i.e., parallel movement of all levels, is roughly $50 \%$ of this.

\section{MAGNETIC MOMENTS AND ENERGIES OF COLLINEAR, COMMENSURATE SDWS IN THE PARENT COMPOUNDS}

For LaFeAsO, our LMTO calculations with imposed FM order converge to a nonmagnetic solution. For stripe and checkerboard AFM orders we calculate magnetic stabilization energies of, respectively, 78 and $39 \mathrm{meV} / \mathrm{Fe}$, values which compare well with those, 84 and $26 \mathrm{meV} / \mathrm{Fe}$, obtained from LAPW calculations for the experimental structure ${ }^{22}$ Our sublattice magnetizations (Fe moments) of 1.3 for stripe and $1.2 \mu_{\mathrm{B}} /$ Fe for checkerboard AFM order are somewhat smaller than those, 1.8 and $1.5 \mu_{\mathrm{B}} / \mathrm{Fe}$, found with LAPW 22 This could be due to integrating the spin-density over different regions, i.e., LMTO and LAPW spheres have different sizes. The LSDA band structure and Fermi surface for stripe order are in a good agreement with those obtained with LAPW 13 Finally, we mention that without the downwards $\epsilon_{\nu x y}$ shift, 
LMTO yields slightly larger energies, 95 and $53 \mathrm{meV} / \mathrm{Fe}$, and moments, 1.4 and $1.4 \mu_{\mathrm{B}} / \mathrm{Fe}$.

For $\mathrm{BaFe}_{2} \mathrm{As}_{2}$, we also find no FM solution. For stripe order, we find a somewhat lower stabilization energy, 62 $\mathrm{meV} / \mathrm{Fe}$, than for $\mathrm{LaFeAsO}$ but the same moment, 1.3 $\mu_{\mathrm{B}} /$ Fe. Using LAPW (Ref 29) instead of LMTO, we find the same stabilization energy, $85 \mathrm{meV} / \mathrm{Fe}$, as for LaFeAsO, and a slightly smaller moment, $1.7 \mu_{\mathrm{B}} / \mathrm{Fe}$. The calculated LMTO and LAPW band structures for the striped phase agree reasonably well. LMTO calculations performed for the experimental low-temperature orthorhombic structure $\underline{\underline{\underline{6}}}$ with the FM stripes oriented either along the longer or the shorter axis result in a lower total energy for the latter. This is in accord with the experimental data in Ref. 9 and a previous calculation. ${ }^{15}$

For stripes in both $\mathrm{LaFeAsO}$ and $\mathrm{BaFe}_{2} \mathrm{As}_{2}$, the maximum exchange splitting, i.e., that of degenerate bands, $\varepsilon_{j \mathbf{k}}$ and $\varepsilon_{j^{\prime} \mathbf{k}+\mathbf{q}}$, with the same dominant $d$ character, ${ }^{26}$ is $\Delta \approx 1.2 \mathrm{eV}$, and this is consistent with an Fe moment of $M \approx 1.3 \mu_{\mathrm{B}} / \mathrm{Fe}$ and Stoner theory: $\Delta=M I$, with the usual value of the Fe effective Stoner parameter $(\sim$ Hunds rule $\left.J_{H}\right): I \approx 0.9 \mathrm{eV}: \underline{30,31}$ Since $\Delta$ is an order of magnitude larger than the energies of the (paramagnetic, un-coupled) electron and hole pockets with respect to the Fermi energy, but considerably smaller that the sub-band widths - which are several $\mathrm{eV}$ - the strength of the LSDA exchange coupling is intermediate when the magnetic order is stripe or checkerboard. This means that neither Fermi surface nesting, nor the difference between the band structures of pure $\mathrm{LaFeAsO}$ and pure $\mathrm{BaFe}_{2} \mathrm{As}_{2}$, nor the $150 \mathrm{meV}$ adjustment for LaFeAsO, strongly affects the magnetic moments and energies.

Although the spin-orbit coupling in the $\mathrm{Fe} 3 d$ shell is weak $\left(\xi_{\mathrm{Fe} 3 d} \approx 60 \mathrm{meV}\right)$, it does lead to a distinct magnetocrystalline anisotropy with the Fe moment lying in the plane and perpendicular to the FM stripes. This has been shown by neutron diffraction for $\mathrm{BaFe}_{2} \mathrm{As}_{2}, \underline{\underline{9}}$ Our relativistic LMTO calculations agree with this: For $\mathrm{LaFeAsO}$ we find that it costs $0.27 \mathrm{meV} / \mathrm{Fe}$ to turn the moment in the plane from the easy to the FM-stripe direction, and an additional $0.13 \mathrm{meV} / \mathrm{Fe}$ to turn the moment perpendicular to the plane. For $\mathrm{BaFe}_{2} \mathrm{As}_{2}$, the corresponding energies are 0.16 and $0.04 \mathrm{meV} / \mathrm{Fe}$, i.e., the anisotropies are smaller.

\section{MAGNETIC MOMENTS AND ENERGIES OF SPIN SPIRALS IN THE DOPED COMPOUNDS}

\section{A. Spin spirals}

A spin spiral is characterized by the following properties: Upon a lattice translation $\mathbf{t}$, the magnitude of the magnetization density and its projection onto a global $z$ direction are unchanged, but the projection onto the perpendicular $(x, y)$ plane rotates by an angle $\varphi(\mathbf{t})$ proportional to the projection of the translation onto the wave vector $\mathbf{q}$ of the spin spiral. The magnetization-density of a spin spiral thus satisfies the equation

$$
\begin{aligned}
& \mathbf{M}(\mathbf{r}+\mathbf{t}, \mathbf{q})=\mathbf{M}(\mathbf{r}, \mathbf{q}) \\
& \quad \cdot\{\hat{\mathbf{z}} \hat{\mathbf{z}} \cos \theta+[\hat{\mathbf{x}} \hat{\mathbf{x}} \cos \varphi(\mathbf{t})+\hat{\mathbf{y}} \hat{\mathbf{y}} \sin \varphi(\mathbf{t})] \sin \theta\} \\
& \text { with } \varphi(\mathbf{t})=\pi \mathbf{q} \cdot \mathbf{t} .
\end{aligned}
$$

(The factor $\pi$ merely follows from the convention chosen in Sec. IIIf for $\mathbf{t}$ referring to the Fe sublattice.) Examples of such spin spirals are shown by the solid arrows in Fig. 4 at the bottom of the right-hand side. Note that for $\mathrm{Fe}$ atoms lying along rows perpendicular to $\mathbf{q}$, the alignment is FM.

In order to solve the one-electron problem in the presence of such a spin spiral, one may span the one-electron Hilbert space by a complete set of localized orbitals, $\phi_{j}(\mathbf{r}-\mathbf{t})$, times pure spin-functions, $\chi_{\mathbf{t}}(\sigma)=\alpha_{\mathbf{t}}(\sigma)$ or $\beta_{\mathbf{t}}(\sigma)$, whose quantization direction is chosen along the local direction of the magnetization. In this representation, the one-electron Hamiltonian without spinorbit coupling is translationally invariant, albeit with qdependent hopping integrals, so that there is no coupling between Bloch sums, $\sum_{\mathbf{t}} \phi_{j}(\mathbf{r}-\mathbf{t}) \chi_{\mathbf{t}}(\sigma) \exp (\pi i \mathbf{k} \cdot \mathbf{t})$, with different Bloch vectors. As a consequence, the oneelectron problem can be solved for any $\mathbf{q}$, without increasing the size of the primitive cell, provided that spin-orbit coupling is neglected ${ }^{32}$ This, together with the LSDA, enables simple calculation of spin-spiral moments and total energies.

In our LMTO calculations, the localized orbitals were taken to be the partial waves truncated outside their sphere. This means that we forced the direction of magnetization to be constant inside each sphere. The moment that we quote is the one integrated over an $\mathrm{Fe}$ sphere. We considered spin spirals for which the magnetization is in the $\mathrm{Fe}(x, y)$ plane, i.e., $\theta=\pi / 2$ and first took $\mathbf{q}$ to lie in the plane and $\mathbf{t}$ to span the square $\mathrm{Fe}$ sublattice. To achieve this in calculations employing the tetragonal translational cell, the phases $\varphi_{i}$, which determine the magnetization directions in two Fe spheres at positions $\boldsymbol{\tau}_{i}$, were fixed to $\varphi_{i}=\mathbf{q} \cdot \boldsymbol{\tau}_{i}$.

The spin spiral with $\mathbf{q}=\bar{\Gamma}(0,0)$ produces FM order. In the spin spiral with $\mathbf{q}=\overline{\mathrm{X}}(1,0)$, the moments rotates by $\pi$ upon translation by $\mathbf{t}=(1,0)$, and by 0 upon translation by $\mathbf{t}=(0,1)$, i.e., the order is stripe with AFM alignment of nearest-neighbor moments along the $x$ direction and FM alignment along the $y$ direction. In the spin spiral with $\mathbf{q}=\overline{\mathrm{M}}(1,1)$, the moments rotate by $\pi$ upon translation by $\mathbf{t}=(1,0)$, as well as by $\mathbf{t}=(0,1)$, i.e., the order is checkerboard with all four nearest-neighbor moments antiparallel and all four second-nearest moments parallel. These spin spirals with $\mathbf{q}$ at high-symmetry points are all collinear and commensurate.

Noncollinear and incommensurate spin spirals with $\mathbf{q}$ near -but not at- $\overline{\mathrm{X}}$ and $\overline{\mathrm{M}}$ are illustrated at the bottom of the right-hand side of Fig. 4 When $\mathbf{q}$ is on the $\bar{\Gamma} \overline{\mathrm{X}}$ line, the order along the $y$ direction remains FM, while going from one $\mathrm{Fe}$ to the next in the positive $x$ direction, the moment rotates by between 0 and $\pi$. When 

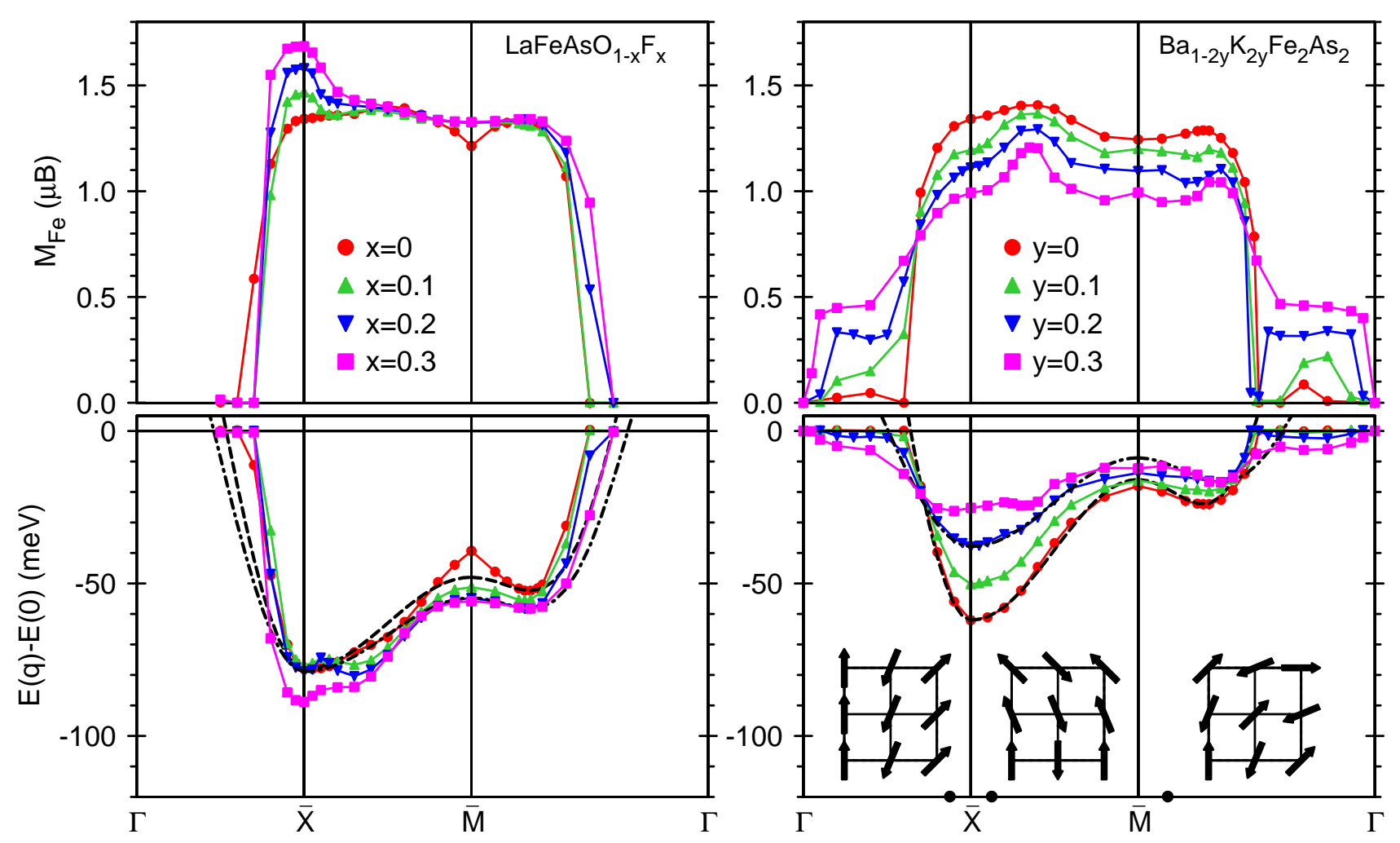

FIG. 4: (Color online) Magnetic moments (upper panels) and energies (lower panels) per Fe of in-plane spin spirals as functions of $\mathbf{q}=\left(q_{x}, q_{y}, 0\right)$ along the boundaries $\bar{\Gamma}(0,0)-\overline{\mathrm{X}}(1,0)-\mathrm{M}(\overline{1}, 1)-\bar{\Gamma}(0,0)$ of the irreducible part of the large BZ for different electron $(x)$ and hole $(y)$ dopings. The energies of the $j_{1}-j_{2}$ Heisenberg model for $x(y)=0$ and 0.2 are given by, respectively, dashed and dash-dotted lines. Representative real-space spin structures are shown at the bottom right for the $\mathbf{q}$ vectors denoted by dots.

$\mathbf{q}$ is on the $\overline{\mathrm{X}} \overline{\mathrm{M}}$ line, the order along the $x$ direction is AFM, while upon going from one Fe to the next in the positive $y$ direction, the moment rotates by between 0 and $\pi$. When finally $\mathbf{q}$ is on the $\bar{M} \bar{\Gamma}$ line, the moment rotates by the same angle, lying between 0 and $\pi$, regardless of whether $\mathbf{t}=(1,0)$ or $(0,1)$, and the order along the second-nearest-neighbor direction perpendicular to $\mathbf{q}$, i.e., for $\mathbf{t}=(1,-1)$, is FM.

We now discuss the calculated results shown in this figure for the magnetic moments (top) and energies (bottom) of spin spirals in electron-(left) and hole-doped (right) compounds as functions of $\mathbf{q}$ along the triangular boundary $\bar{\Gamma}-\overline{\mathrm{X}}-\overline{\mathrm{M}}-\bar{\Gamma}$ of the irreducible part of the large BZ (see Fig. 1).

\section{B. Pure and electron-doped $\mathrm{LaFeAsO}_{1-x} \mathbf{F}_{x}$}

For undoped $\mathrm{LaFeAsO}$, i.e. for $x=0$ (red dots), the lowest energy is reached at the $\overline{\mathrm{X}}$ point, i.e., for stripe AFM order. This agrees with the results of previous calculations $\frac{14,15,20}{20}$ and experimental data $\stackrel{10}{=}$ When moving from $\overline{\mathrm{X}}(1,0)$ toward $\bar{\Gamma}(0,0)$, the AFM order between nearest-neighbor Fe rows along $y$ is destroyed, and this leads to a rapid decrease in moment and increase in energy; for $|\mathbf{q}| \equiv q<0.6$ the self-consistent solution is nonmagnetic. When moving from $\overline{\mathrm{X}}(1,0)$ toward $\overline{\mathrm{M}}(1,1)$, the FM order in the Fe rows along $y$ is destroyed and becomes AFM at $\bar{M}$. Whereas the moment at first remains nearly constant at $1.4 \mu_{\mathrm{B}}$, but finally decreases to 1.2 $\mu_{\mathrm{B}}$ at $\overline{\mathrm{M}}$, the energy increases nearly parabolically from -78 to $-39 \mathrm{meV}$. Moving from $\overline{\mathrm{M}}(1,1)$ toward $\bar{\Gamma}(0,0)$, the nearest-neighbor AFM order develops toward FM order by preserving the FM order between second-nearest neighbors along $\mathbf{t}=(1,-1)$ but destroying the one along $\mathbf{t}=(1,1)$. Hereby the moment first increases slightly, but then decreases rapidly and vanishes when $q<0.7$. The energy falls to a local minimum at $q \approx 1$, and then increases rapidly for $q$ decreasing to 0.7 where the moment disappears.

As shown by the dashed curve in Fig. 4, for $q \gtrsim 0.7$ our calculated $E(\mathbf{q})$ for undoped LaFeAsO is approximated reasonably well by a classical Heisenberg model on the square lattice with AFM exchange coupling constants $j_{1}$ and $j_{2}$ between, respectively, nearest and next-nearest neighbors. We obtain the ratio $j_{2} / j_{1}=0.71(>1 / 2)$ by fitting to the q position of the local minimum along $\overline{\mathrm{M}} \bar{\Gamma}$ and then obtain the values $j_{1}=81$ and $j_{2}=57 \mathrm{meV}$ 
by fitting the energy difference $E_{\min }(\overline{\mathrm{M}} \bar{\Gamma})-E(\overline{\mathrm{X}})$ and the value $S \equiv \frac{1}{2} M(\overline{\mathrm{X}})=0.67$. The $\mathbf{q}$-independent constant is finally chosen such that the Heisenberg model fits the calculated energy at $\overline{\mathrm{X}}$ (and the minimum along $\bar{M} \bar{\Gamma}$ ). Our values of $j_{1}$ and $j_{2}$ are comparable to those obtained from Fig. 3 in Ref. 14 when interpolated to $M(\overline{\mathrm{X}})=1.3 \mu_{\mathrm{B}}$, although our $j_{2} / j_{1}$ is somewhat higher. The anisotropic exchange coupling constants calculated in Ref. 13 are meaningful only for small deviations from stripe AFM order, that is for $\mathbf{q}$ near $\overline{\mathrm{X}}$, and cannot be directly compared with our effective $j_{1}$ and $j_{2}$. Nevertheless, our $j_{1} S^{2}=420 \mathrm{~K}$ and $j_{2} S^{2}=297 \mathrm{~K}$ are of the same order of magnitude as $-J_{1}^{\perp}=550 \mathrm{~K}$ and $-J_{2}^{\perp}=260$ $\mathrm{K}$ of Ref. 13. This indicates that the three different approaches result in the comparable exchange interactions. Although the overall shape of the dashed $E(\mathbf{q})$ curve given by the Heisenberg model is similar to the red-dotted one obtained from our LSDA calculation, discrepancies can be clearly seen even in the part of the BZ where the Fe moment remains nearly constant: The calculated energy is higher in the vicinity of the $\overline{\mathrm{M}}$ point and it increases far more rapidly when going from $\overline{\mathrm{X}}$ toward $\bar{\Gamma}$.

Our calculations reveal that a new local minimum of $E(\mathbf{q})$ develops along $\overline{\mathrm{X}} \overline{\mathrm{M}}$ near $(1,0.3)$ when the electron doping exceeds about $5 \%$. For $0.10<x<0.25$, this minimum is deeper than the one a $\overline{\mathrm{X}}$, i.e. it is the global minimum, and this means that stripe order is unstable against formation of an incommensurate noncollinear SDW. This is in line with experimental phase diagrams for $\mathrm{REFeAsO} \mathrm{O}_{1-x} \mathrm{~F}_{x}$ compounds which show that magnetic order is rapidly suppressed by $\mathrm{F}$ doping $\underline{\underline{11}}$ For $x=0.3$ we find that the energy minimum has returned to $\overline{\mathrm{X}}$, although a well-defined shoulder can still be seen at $(1,0.3)$. This instability toward an incommensurate SDW cannot be reproduced by fitting to the $j_{1}-j_{2}$ Heisenberg model, as is clearly seen by comparison of the dotdashed and blue $\mathbf{\nabla}$ curves.

Electron doping is seen to increase the moment for stripe AFM order from 1.4 to $1.7 \mu_{\mathrm{B}}$ for $x=0.3$. But this increase is localized to $\mathbf{q}$ being close to $\overline{\mathrm{X}}$.

The destabilization of stripe AFM order with electron doping seems to be caused by occupation of a narrow peak of the density of states (DOS), which in undoped $\mathrm{LaFeAsO}$ is $\sim 150 \mathrm{meV}$ above the Fermi level $\left(\varepsilon_{F}\right)$. The band responsible for this peak is the paramagnetic Fe $d_{y z}$ band which hardly disperses in the $k_{x}$ direction and stays within $\pm 200 \mathrm{meV}$ of the Fermi level over a region of $\mathbf{k}$ space near the entire $\bar{M} \bar{Y}$ line. This is the band seen in Fig. 2 to connect the $d_{y z}$-like hole pocket at $\bar{M}$ with the superellipsoidal electron pocket at $\bar{Y}$. Now, FM stripes in the $y$ direction with AFM order along $x$ will couple states at $\mathbf{k}$ with those at $\mathbf{k}+\mathbf{q}=\mathbf{k}+(1,0)$, i.e. will fold the large $\mathrm{BZ}$ perpendicular to the $y$ direction, thus placing $\overline{\mathrm{M}}$ on top of $\bar{Y}$, and exchange-split states with the same $\mathrm{Fe}$ $d$ character by $\sim \pm 0.5 \mathrm{eV}$. As a result, the flat $d_{y z}$ band will have its upper minority-spin $d_{y z}$ band $\sim 150 \mathrm{meV}$ above $\varepsilon_{F}$ in the undoped compound $\underline{26}$ Since the DOS near $\varepsilon_{F}$ is very low in the stripe-ordered undoped compound, not much electron doping is needed to occupy part of the flat $d_{y z}$ minority-spin band. For q moving away from $\overline{\mathrm{X}}$ in the perpendicular direction, i.e., toward $\bar{M}$, the DOS peak soon splits in two. The concomitant decrease in band energy for dopings such that $\varepsilon_{F}$ is in the valley between the subpeaks compensates for the decrease in negative magnetic exchange energy $\left(-\frac{1}{4} M^{2} I\right)$ caused by the decrease in magnetization seen in the upper left-hand side of Fig. 4. The magnetization decreases because the nesting is less good (less phase space available for gapping) when $\mathbf{q}$ moves so far away from $\overline{\mathrm{X}}$ in the perpendicular direction that $\mathbf{q}$ does not so well translate the $\mathbf{k}$ tube around $\bar{M} \bar{Y}$ in which the $d_{y z}$ band is flat onto itself. The energy minimum finally shifts back to $\mathbf{q}=\mathrm{X}(\overline{1}, 0)$ once the electron doping is so high $(>25 \%)$ that the narrow DOS peak is completely filled, i.e., when $\varepsilon_{F}$ is above the flat part of the minority-spin $d_{y z}$ band.

Recently, a commensurate-to-incommensurate transition with increasing $x$ in $\mathrm{LaFeAsO}_{1-x} \mathrm{~F}_{x}$ has been reported in Ref. 33 on the base of full-potential LAPW spin-spiral calculations. These calculations were however performed using calculated As positions and can therefore not be compared directly our calculations based on the experimental structure.

Returning now to the undoped compound and $\mathbf{q}$ moving away from $\overline{\mathrm{X}}$ toward $\overline{\mathrm{M}}, \varepsilon_{F}$ is below both subpeaks and the Heisenberg-type dependence of $E(\mathbf{q})$ persists until in the vicinity of $\bar{M}$ a huge DOS peak appears just below $\varepsilon_{F}$.

For stripe-ordered pure $\mathrm{LaFeAsO}$, another narrow DOS peak exists $270 \mathrm{meV}$ above $\varepsilon_{F}$ and arises from the upper minority-spin $d_{x y}$-like band being split from the lower minority-spin band due to folding of the $\overline{\mathrm{M}}$-centered hole pocket onto the $\overline{\mathrm{Y}}$-centered electron pocket ${ }^{26}$

We estimated the strength of the interlayer exchange coupling by performing calculations for spin spirals with $\mathbf{q}$ not lying in the Fe plane. Specifically we took $\mathbf{q}=$ $\left(1,0, q_{z}\right)$ corresponding to stripe order in each layer and rotation of the magnetic moments between layers. These calculations resulted in a very small change in energy when changing the alignment of the Fe moments along the $c$ direction from ferromagnetic to antiferromagnetic. A weak dependence of the energy on the magnetic order along the $c$ axis was also reported in Ref. 20. This nearly two-dimensional character of the magnetic interactions is not affected by $\mathrm{F}$ doping.

The $\mathbf{q}$ dependencies of the spin-spiral moment and energy calculated for $\mathrm{LaFeAsO}_{1-x} \mathrm{~F}_{x}$ without downshifting the $d_{x y}$ energies are shown in Fig. 5. Also in this case is the energy minimum of undoped $\mathrm{LaFeAsO}$ at $\overline{\mathrm{X}}$, but $\sim 20$ meV deeper. The local minimum along $\bar{M} \bar{\Gamma}$ is at the same q position, but merely $10 \mathrm{meV}$ deeper, so that the fitted values, $j_{1}=93$ and $j_{2}=66 \mathrm{meV}$, are a bit larger but have the same ratio. For electron-doped $\mathrm{LaFeAsO}_{1-x} \mathrm{~F}_{x}$, the $\mathbf{q}$ dependence of the energy is somewhat stronger and exhibits a shoulder at $\mathbf{q} \approx(1,0.3)$ which does not develop into a well-defined minimum. Nevertheless, destabiliza- 


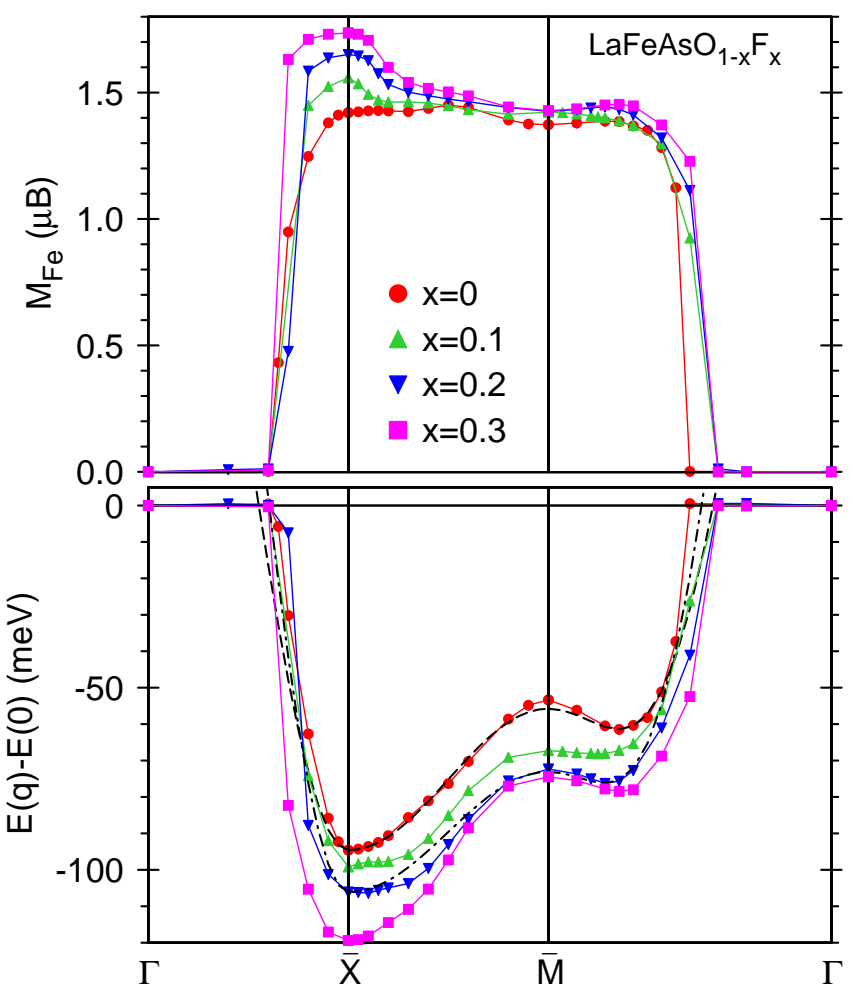

FIG. 5: (Color online) Same as Fig. 4 but for $\mathrm{LaFeAsO}_{1-x} \mathrm{~F}_{x}$ without downshifting the Fe $d_{x y}$ energies.

TABLE II: The doping dependence of the Fe magnetic moments $M(\overline{\mathrm{X}})$ calculated for stripe $\mathrm{AF}$ and the exchange coupling constants $j_{1}$ and $j_{2}$ in $\mathrm{LaFeAsO}_{1-x} \mathrm{~F}_{x}$ and $\mathrm{Ba}_{1-2 y} \mathrm{~K}_{2 y} \mathrm{Fe}_{2} \mathrm{As}_{2}$. For $\mathrm{LaFeAsO}_{1-x} \mathrm{~F}_{x}$, the values calculated both with and without the shift of the $d_{x y}$ states are presented. The latter are given in parentheses.

\begin{tabular}{|c|c|c|c|c|c|}
\hline Compound & $x / y$ & $M(\overline{\mathrm{X}})\left(\mu_{\mathrm{B}}\right)$ & $j_{2} / j_{1}$ & $j_{1}(\mathrm{meV})$ & $j_{2}(\mathrm{meV})$ \\
\hline \multirow[t]{7}{*}{$\mathrm{LaFeAsO}_{1-x} \mathrm{~F}_{x}$} & 0.3 & $\begin{array}{c}1.68 \\
(1.74)\end{array}$ & $\begin{array}{c}0.71 \\
(0.71)\end{array}$ & $\begin{array}{c}61 \\
(77)\end{array}$ & $\begin{array}{c}43 \\
(54)\end{array}$ \\
\hline & 0.2 & 1.58 & 0.71 & 47 & 33 \\
\hline & & $(1.65)$ & $(0.62)$ & (103) & (63) \\
\hline & 0.1 & 1.47 & 0.66 & 71 & 47 \\
\hline & & $(1.56)$ & $(0.59)$ & $(160)$ & (94) \\
\hline & 0 & 1.34 & 0.71 & 81 & 57 \\
\hline & & $(1.42)$ & $(0.71)$ & $(93)$ & $(66)$ \\
\hline \multirow[t]{4}{*}{$\mathrm{Ba}_{1-2 y} \mathrm{~K}_{2 y} \mathrm{Fe}_{2} \mathrm{As}_{2}$} & 0 & 1.34 & 0.77 & 95 & 73 \\
\hline & 0.1 & 1.19 & 0.85 & 77 & 65 \\
\hline & 0.2 & 1.11 & 1.10 & 39 & 43 \\
\hline & 0.3 & 0.99 & 1.10 & 19 & 21 \\
\hline
\end{tabular}

tion of the commensurate collinear stripe order does occur, although the details are seen to depend sensitively on the underlying band structure.

\section{Pure and hole-doped $\mathrm{Ba}_{1-2 y} \mathrm{~K}_{2 y} \mathrm{Fe}_{2} \mathrm{As}_{2}$}

The spin-spiral moments and energies as functions of $\mathbf{q}=\left(q_{x}, q_{y}, 0\right)$ for $\mathrm{Ba}_{1-2 y} \mathrm{~K}_{2 y} \mathrm{Fe}_{2} \mathrm{As}_{2}$ with $y=0,0.1,0.2$, and 0.3 holes per FeAs are shown in the right-hand panel of Fig. 4 For undoped $\mathrm{BaFe}_{2} \mathrm{As}_{2}$, these curves (red dots) are qualitatively similar to those calculated for $\mathrm{LaFeAsO}$, and the trend for increasing hole-doping of $\mathrm{Ba}_{1-2 y} \mathrm{~K}_{2 y} \mathrm{Fe}_{2} \mathrm{As}_{2}$ continues the trend for decreasing electron-doping of $\mathrm{LaFeAsO}_{1-x} \mathrm{~F}_{x}$, i.e., $y \sim-x$. The calculated energy gain $E(\bar{\Gamma})-E(\overline{\mathrm{X}})$ due to the formation of stripe AFM order in $\mathrm{BaFe}_{2} \mathrm{As}_{2}$ is somewhat smaller than in LaFeAsO, but the energy difference $E_{\min }(\overline{\mathrm{M}} \bar{\Gamma})-E(\overline{\mathrm{X}})$ is a bit larger and this causes the fitted values $j_{1}=95$ and $j_{2}=73 \mathrm{meV}$ to be a bit larger. The ratio $j_{2} / j_{1}=0.77$ is slightly larger than for LaFeAsO. Hole doping strongly reduces the stripe-formation energy, $E(\bar{\Gamma})-E(\overline{\mathrm{X}})$. Nevertheless, the energy minimum remains at $\bar{X}$ until the hole doping exceeds $25 \%$, at which point the minimum splits in two with the lowest lying along $\overline{\mathrm{X}} \bar{\Gamma}$. Our calculations thus show that stripe order is more resistant to hole doping in $\mathrm{Ba}_{1-2 y} \mathrm{~K}_{2 y} \mathrm{Fe}_{2} \mathrm{As}_{2}$ than to electron doping in $\mathrm{LaFeAsO}_{1-x} \mathrm{~F}_{x}$. This conclusion is supported by experimental observations of the traces of the spin-densitywave phase for K-doping as high as 0.4, i.e., well into the superconducting region $, 8,12$

The local minimum along $\bar{M} \bar{\Gamma}$ moves toward $\bar{\Gamma}$ as hole doping increases. As a consequence, the estimated $j_{2} / j_{1}$ increases and reaches the value of 1.1 when $y=0.2$. Since the energy difference $E_{\min }(\overline{\mathrm{M}} \bar{\Gamma})-E(\overline{\mathrm{X}})$ decreases with hole doping, the values of the effective coupling constants decrease to $j_{1}=39$ and $43 \mathrm{meV}$ when $y=0.2$.

The Fe moment calculated for stripe AFM order decreases from $1.34 \mu_{\mathrm{B}}$ in the undoped compound to 0.99 $\mu_{\mathrm{B}}$ for $y=0.3$. The maximum of $M(\mathbf{q})$ is however not at $\overline{\mathrm{X}}$, but at $\mathbf{q} \approx(1,0.4)$ along $\overline{\mathrm{X}} \overline{\mathrm{M}}$. This maximum becomes more pronounced with hole doping. In contrast to the situation in $\mathrm{LaFeAsO}_{1-x} \mathrm{~F}_{x}$, where for $\mathbf{q}$ in a large region around $\bar{\Gamma}$ the non-magnetic solution is stable, in $\mathrm{Ba}_{1-2 y} \mathrm{~K}_{2 y} \mathrm{Fe}_{2} \mathrm{As}_{2}$ magnetic solutions exist closer and closer to $\bar{\Gamma}$ and with increasing moment as the hole doping increases. For $y=0.3$ the moment is nearly 0.5 $\mu_{B}$, except very close to $\bar{\Gamma}$. A FM solution is thus being approached.

Calculations for spirals with non-zero $q_{z}$ reveal much stronger dependence of the energy on the relative orientation of the Fe moments in adjacent FeAs layers than in LaFeAsO. This is due to the stronger $k_{z}$ dispersion of the As- $p_{z}$ hybridized bands which was discussed in Sec. III. In $\mathrm{BaFe}_{2} \mathrm{As}_{2}$, inter-layer nearest As neighbors are on top of each other and we find that the lowest-energy solution is for nearest-neighbor layers having parallel AFMordered stripes. The energy for FM ordering between parallel stripes is $4 \mathrm{meV} / \mathrm{Fe}$ higher, and the energies for orthogonal stripes are intermediate. This is in accord with the experimental observations ${ }^{9}$ and results obtained from calculations for collinear spin arrangements $\underline{\underline{19}}$ 


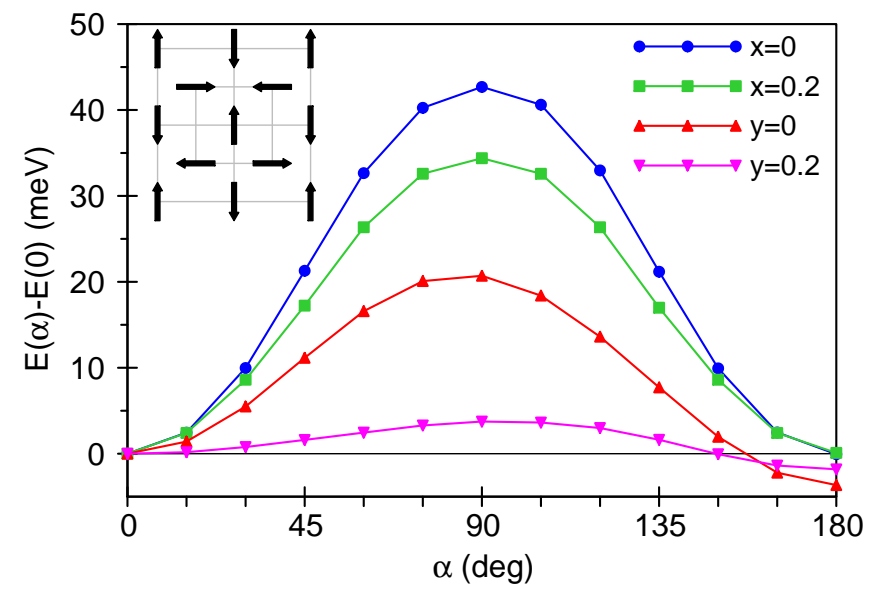

FIG. 6: (Color online) Dependences of the energy of $\mathrm{LaFeAsO}_{1-x} \mathrm{~F}_{x}$ and $\mathrm{Ba}_{1-2 y} \mathrm{~K}_{2 y} \mathrm{Fe}_{2} \mathrm{As}_{2}$ on the angle $\alpha$ between the moments of Fe nearest neighbors. The inset (turned $45^{\circ}$ with respect to the one in Fig. 4) shows the spin arrangement in the Fe plane for $\alpha=90^{\circ}$

\section{Applicability of the simple $j_{1}-j_{2}$ Heisenberg model}

The values of the stripe-ordered moment, $M(\overline{\mathrm{X}})$, the $j_{2} / j_{1}$ ratio determined by the position of the local minimum along $\bar{M} \bar{\Gamma}$, as well as the values of $j_{1}$ and $j_{2}$ are collected in Table Ifor all electron and hole dopings considered. Whereas $M(\overline{\mathrm{X}})$ and $j_{2} / j_{1}$ exhibit clear trends with doping and position of the $d_{x y}$ band, $j_{1}$ and $j_{2}$ scatter more. This enforces a conclusion that an incommensurable SDW emerging upon electron doping is a typical band-structure effect: The magnetic energies of the doped compounds can hardly be described by the simple $j_{1}-j_{2}$ Heisenberg model.

In order to test the applicability of $j_{1}-j_{2}$ Heisenberg model for merely the parent compounds, we then performed calculations for spin structures with $\mathbf{q}=(1,0)$, but with the phases $\varphi_{i}$ for the two Fe sites in the tetragonal unit cell chosen in such a way that the angle, $\alpha=\varphi_{1}-\varphi_{2}$, between their magnetic moments varied from 0 to $180^{\circ}$. As seen from the inset in Fig. 6] which is turned $45^{\circ}$ with respect to the one in Fig. 4 the Fe sites form two interpenetrating square sublattices. At $\mathbf{q}=(1,0)$, Fe moments in each sublattice are ordered antiferromagnetically as shown in the inset. The angles between the moment of an Fe and those on the two pairs of nearest neighbors, belonging to the other sublattice, are respectively $\alpha$ and $\pi-\alpha$ and, hence, the Heisenberg energy is independent of $\alpha$. The calculated dependencies of the energy on $\alpha$ are shown in Fig. 6. The spin arrangement corresponding to $\alpha=180^{\circ}$ is exactly the same as the one generated by the spiral with $\mathbf{q}=(0,1)$ and $\alpha=0$. In other words, the spirals with $\alpha=0$ and $\alpha=180^{\circ}$ result in stripe order with the AFM chains parallel to $x$ and $y$ axis, respectively. The corresponding solutions for
LaFeAsO are degenerate. In $\mathrm{BaFe}_{2} \mathrm{As}_{2}$ with the bodycentered unit cell the variation in $\alpha$ is accompanied by a change of angle between the Fe moments in the adjacent FeAs layers. Due to the AFM alignment of the Fe moments along the $c$ axis, the solution with $\alpha=180^{\circ}$ has a lower energy than that with $\alpha=0$ having FM order along c.

Our LSDA calculations show that for both compounds the energy depends strongly on the relative orientation of the Fe moments, with $E(\alpha=90)-E(\alpha=0)$ being comparable to the energy difference between the two collinear AFM solutions with $\mathbf{q}=(1,0)$ and $\mathbf{q}=(1,1)$ discussed in connection with Fig. 4. The calculated results behave like $E(\alpha)=C \sin ^{2} \alpha$, which does not appear in the $j_{1}-j_{2}$ Heisenberg model but can be recovered by adding a biquadratic term proportional to $\left(\mathbf{S}_{i} \cdot \mathbf{S}_{j}\right)^{2}$, with $\mathbf{S}_{i}$ and $\mathbf{S}_{j}$ being spins on Fe nearest neighbors.

The strong dependence of the energy on the relative orientation of the two AFM Fe sublattices points to the non-Heisenberg character of the interactions between $\mathrm{Fe}$ moments even in undoped $\mathrm{LaFeAsO}$ and $\mathrm{BaFe}_{2} \mathrm{As}_{2}$. The $E(\alpha)$ curves calculated for doped compounds (Fig. 6) show a similar, although weaker, $\alpha$ dependence, especially for $\mathrm{Ba}_{0.6} \mathrm{~K}_{0.4} \mathrm{Fe}_{2} \mathrm{As}_{2}$.

Thus, among the variety of spin configurations, which were degenerate in the $j_{1}-j_{2}$ Heisenberg model, a collinear spin arrangement with either $\mathbf{q}=(1,0)$ or $\mathbf{q}=(0,1)$ is favored already at the level of the LSDA electronic structure. Such a magnetically ordered solutions lower the symmetry of the lattice from tetragonal to orthorhombic and lifts the degeneracy of Fe $d_{y z}$ and $d_{z x}$ states. This symmetry lowering is apparently responsible for the anisotropy of the exchange interactions calculated for stripe AFM order in Ref. 13 .

\section{NONINTERACTING SUSCEPTIBILITY}

With an Fe moment of $\sim 1.5 \mu_{\mathrm{B}}$, the exchange splitting between the majority- and minority-spin Fe $d$ states in the LSDA calculations is about $1.3 \mathrm{eV}$, which is as large as the dispersion of the paramagnetic Fe $d_{x y}$ and $d_{y z}$ and $d_{x z}$ bands over a significant part of the BZ, e.g., the $d_{y z}$ band in a tube around $\bar{M} \bar{Y}$. Such a strong magnetic perturbation dramatically changes the band structure and the topology of the Fermi surface. The limit of a weak magnetic perturbation can be analyzed by studying the $\mathbf{q}$ and doping dependencies of the static, noninteracting linear-response susceptibility. Its imaginary part, $\operatorname{Im} \chi_{0}(\mathbf{q})=\operatorname{Im} \chi_{0}(\mathbf{q}, \omega \rightarrow 0) / \omega$, is determined by the shape of and velocities on the Fermi surface (FS) and is a quantitative measure of FS nesting. The real part, $\operatorname{Re} \chi_{0}(\mathbf{q})=\operatorname{Re} \chi_{0}(\mathbf{q}, \omega=0)$, describes the response of the system to an infinitesimally small perturbation. In contrast to $\operatorname{Im} \chi_{0}(\mathbf{q})$, the electronic states in a wide energy range around $E_{F}$ may contribute to $\operatorname{Re} \chi_{0}(\mathbf{q})$. 
In the present work the noninteracting susceptibility

$$
\begin{array}{r}
\chi_{0}(\mathbf{q}, \omega)=-\frac{1}{V} \sum_{\mathbf{k}, n, n^{\prime}} \frac{f_{n^{\prime}}(\mathbf{k}+\mathbf{q})-f_{n}(\mathbf{k})}{\varepsilon_{n^{\prime}}(\mathbf{k}+\mathbf{q})-\varepsilon_{n}(\mathbf{k})+\omega+i \delta} \\
\times\left\langle\mathbf{k}, n\left|e^{-i \mathbf{q} \cdot \mathbf{r}}\right| \mathbf{k}+\mathbf{q}, n^{\prime}\right\rangle\left\langle\mathbf{k}+\mathbf{q}, n^{\prime}\left|e^{i \mathbf{q} \cdot \mathbf{r}}\right| \mathbf{k}, n\right\rangle
\end{array}
$$

was calculated using the linear response expressions given in Ref. 34. Here, $\varepsilon_{n}(\mathbf{k})$ is the energy of the $n$th band and $f_{n}(\mathbf{k})$ is the Fermi function. First, the imaginary part of $\chi_{0}(\mathbf{q}, \omega)$ was calculated in the $\delta \rightarrow 0$ limit. Then, the real part was obtained by using Kramers-Kronig relations. The matrix elements $\left\langle\mathbf{k}+\mathbf{q}, n^{\prime}|\exp (i \mathbf{q r})| \mathbf{k}, n\right\rangle$ of the perturbation were approximated by expanding the exponent inside each Fe sphere in Bessel functions and keeping only the spherically symmetric term proportional to $j_{0}(\mathbf{q r})$. The expressions for the matrix elements were further simplified by using $j_{0}(\mathbf{q r}) \approx 1$. Within this approximation the contribution of each Fe sphere to the matrix element $\left\langle\mathbf{k}+\mathbf{q}, n^{\prime}|\exp (i \mathbf{q r})| \mathbf{k}, n\right\rangle$ is proportional to the overlap integral of the LMTO wave functions $\Psi_{\mathbf{k}, n}$ and $\Psi_{\mathbf{k}+\mathbf{q}, n^{\prime}}$ inside the sphere. In other words, two states contribute to the susceptibility only if they have similar Fe partial-wave character. Although these approximations are valid only at sufficiently small $|\mathbf{q}|$, they do not affect the analysis of susceptibility peaks which may appear due to the FS nesting. More details on calculation of $\chi_{0}(\mathbf{q}, \omega)$ using the LMTO method can be found in Ref. 35.

The contribution of a particular subset of electronic states to $\chi_{0}(\mathbf{q}, \omega)$ can be discerned by calculating the matrix elements of the perturbation with all coefficients of the LMTO wave functions, except those which correspond to the chosen subset, set to zero. Due to the presence of interference terms, such orbitally resolved contributions to the susceptibility are not additive. However, they allow us to discriminate those states which give the dominant contribution to $\chi_{0}(\mathbf{q}, \omega)$.

The susceptibilities of iron pnictides presented below were calculated starting from self-consistent non-spinpolarized electron densities and neglecting spin-orbit coupling. Thus, because of the degeneracy of Bloch states with different spin projections, $\chi_{0}(\mathbf{q}, \omega)$ calculated using Eq. (1) describes the response of the system to a q-dependent spin as well as charge perturbation. A $32 \times 32 \times 32 \mathbf{k}$ mesh in the small tetragonal BZ was used in the calculations.

\section{A. $\mathrm{LaFeAsO}_{1-x} \mathbf{F}_{x}$}

Nesting between the quasi-two-dimensional FS sheets in the iron pnictides were noted already in the earliest electronic-structure calculations. ${ }^{11,16,20}$ The FS cross sections calculated for undoped LaFeAsO with and without the downwards shift of the Fe $d_{x y}$ energy were shown in Fig. 1 and their nesting pointed out in the caption to this figure, as well as in Sec. III.
The imaginary and real parts of the bare static susceptibility calculated for $\mathrm{LaFeAsO}_{1-x} \mathrm{~F}_{x}$ with downshifted $d_{x y}$ energy are shown in the left-hand panel of Fig. 7 . We first discuss the results for the undoped $(x=0)$ compound. In agreement with previous results ${ }^{11,16}$ the maximum of $\operatorname{Re} \chi_{0}(\mathbf{q})$ is found at $\mathbf{q}=\overline{\mathrm{X}}(1,0)$. A peak of the imaginary part is, however, shifted away from $\overline{\mathrm{X}}$ toward $\bar{M}$. Analysis of the partial-wave resolved contributions to the susceptibility shows that the main contribution to $\operatorname{Im} \chi_{0}(\overline{\mathrm{X}})$ comes from nesting of $\overline{\mathrm{M}}$-centered $d_{y z, x z}$-like hole sheet (blue in the left-hand side of Fig. 1) with the $\bar{Y}$-centered electron sheet (green). This also gives the dominant contribution to the maximum of $\operatorname{Re} \chi_{0}(\overline{\mathrm{X}})$. But also the $d_{x y}$ states (red) contribute significantly to $\operatorname{Re} \chi_{0}(\overline{\mathrm{X}})$ although their contribution to $\operatorname{Im} \chi_{0}(\overline{\mathrm{X}})$ nearly vanishes due to the ineffective nesting of the innermost $\bar{\Gamma}$-centered hole sheet with the $\overline{\mathrm{X}}$-centered electron sheet. As $\mathbf{q}$ moves along $\overline{\mathrm{X}} \overline{\mathrm{M}}$, the hole and electron sheets start to touch when $\mathbf{q} \approx(1,0.13)$ and a peak of the $d_{x y}$ contribution to $\operatorname{Im} \chi_{0}(\mathbf{q})$ appears at this nesting vector. This is responsible for the maximum of $\operatorname{Im} \chi_{0}(\mathbf{q})$ along $\bar{X} \bar{M}$. The $d_{x y}$ contribution to $\operatorname{Re} \chi_{0}(\mathbf{q})$ reaches its maximum at the same q. Since the weight of the Fe $d_{3 z^{2}-1}$ and $d_{x^{2}-y^{2}}$ states in the bands crossing $E_{F}$ is very small, they do not contribute to $\operatorname{Im} \chi_{0}(\mathbf{q})$, whereas their contribution to $\operatorname{Re} \chi_{0}(\mathbf{q})$ is almost constant in the whole $\mathbf{q}$ range.

With electron doping, the hole sheets, centered at $\bar{\Gamma}$ and $\overline{\mathrm{M}}$, shrink and the electron sheets, centered at $\overline{\mathrm{X}}$ and $\bar{Y}$, grow. The hole and electron sheets no longer nest for $\mathbf{q}=\overline{\mathrm{X}}$ so that the susceptibility at $\overline{\mathrm{X}}$ decreases rapidly with doping. Instead, peaks develop in both the imaginary and real parts of $\chi_{0}(\mathbf{q})$ for $\mathbf{q}$ along $\bar{X} \bar{M}$ for which the electron and hole sheets touch. This shift of the susceptibility peaks with increasing $x$ correlates with the shift of the minimum of the $E(\mathbf{q})$ curves calculated for spin spirals (Fig. 4). The shift of the $\operatorname{Re} \chi_{0}(\mathbf{q})$ peak along the $\overline{\mathrm{X}} \bar{\Gamma}$ line, accompanied by strong suppression at $\overline{\mathrm{X}}$, was noted in Ref. 11 .

Comparison of $\chi_{0}(\mathbf{q})$ calculated with (Fig. 7) and without (Fig. 8) shifting the Fe $d_{x y}$ energy shows that the susceptibility of undoped LaFeAsO is sensitive to the changes of the FS shown in Fig. 1 caused by the shift. Without the shift, the $\bar{\Gamma}$-centered $d_{x y}$-like hole sheet is larger and nests almost perfectly the $\overline{\mathrm{X}}$-centered electron sheet when $\mathbf{q}=\overline{\mathrm{X}}$. This leads to $\operatorname{Im} \chi_{0}(\overline{\mathrm{X}})$ being the top of a sharp peak with the dominant contribution coming from the $d_{x y}$ states. The $d_{x y}$ contribution to $\operatorname{Re} \chi_{0}(\mathbf{q})$ also has a sharp maximum at $\overline{\mathrm{X}}$. Since even the largest of the two $\bar{M}$-centered $d_{y z, z x}$ hole sheets is smaller than the $\bar{Y}$-centered electron sheet, nesting of these sheets does not contribute to $\operatorname{Im} \chi_{0}(\overline{\mathrm{X}})$. Their contribution increases as $\mathbf{q}$ shifts away from $\overline{\mathrm{X}}$, but remains weaker than the $d_{x y}$ one. Nevertheless, the $d_{y z, z x}$ contribution to $\operatorname{Re} \chi_{0}(\mathbf{q})$ in the vicinity of the $\overline{\mathrm{X}}$ point is comparable to the $d_{x y}$ one. The $\mathbf{q}$ dependence of the imaginary part of susceptibility is strongly affected by the change of FS nesting caused by the shift of the $d_{x y}$ bands, but the behavior of the real part is much more robust. Its maximum does not move 

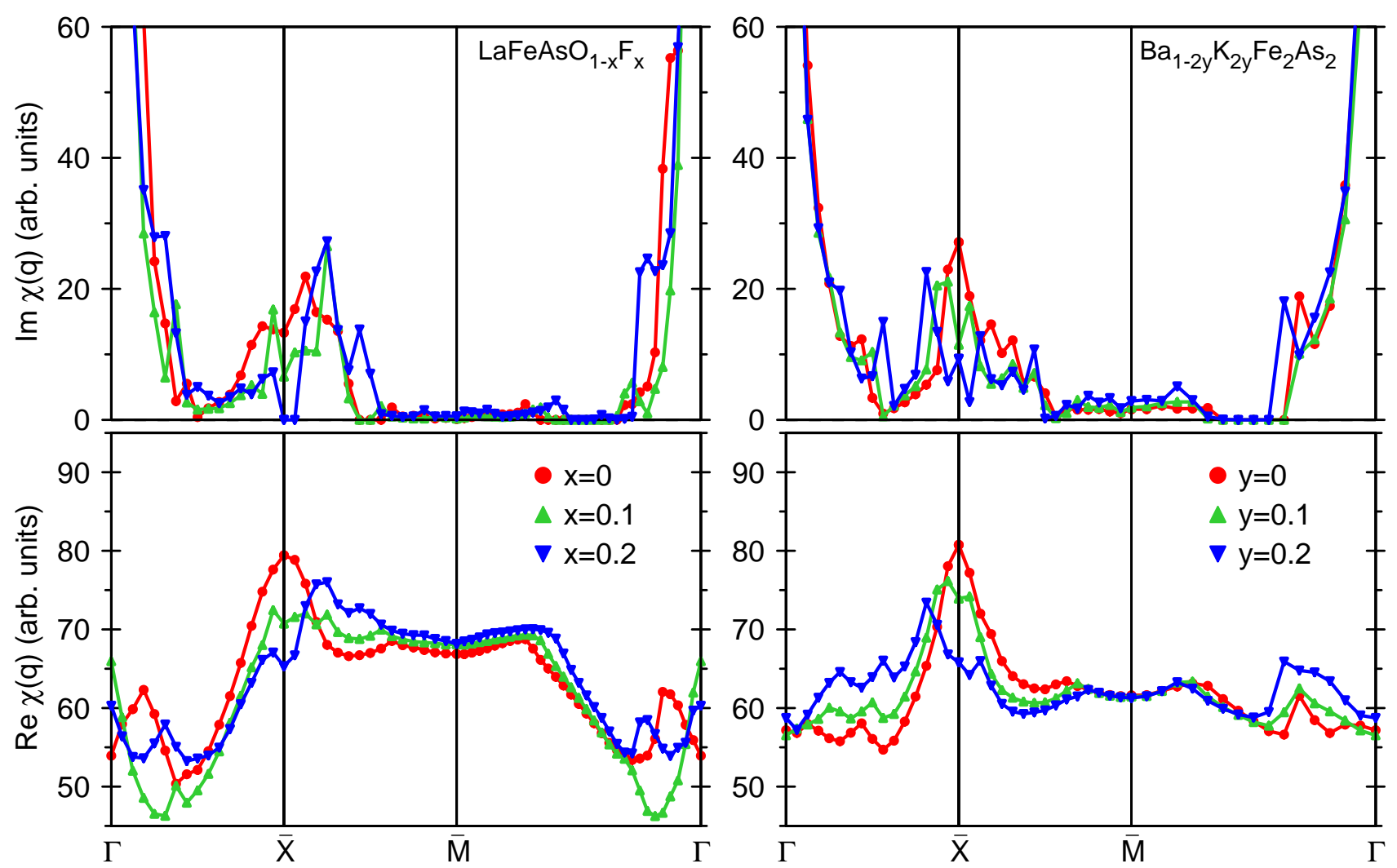

FIG. 7: (Color online) Imaginary and real parts of the bare static susceptibility for $\mathrm{LaFeAsO}_{1-x} \mathrm{~F}_{x}$ and $\mathrm{Ba}_{1-2 y} \mathrm{~K}_{2 y} \mathrm{Fe}_{2} \mathrm{As}_{2}$.

away from the $\overline{\mathrm{X}}$ point but looses its sharpness as the FS nesting becomes less perfect.

The doping dependence of the susceptibility is only weakly affected by the shift of the $d_{x y}$ states. This may be one reason why different layered iron arsenides exhibit similar properties, in spite of the variation in their band structures.

\section{B. $\mathbf{B a}_{1-2 y} \mathbf{K}_{2 y} \mathbf{F e}_{2} \mathbf{A s}_{2}$}

As explained in Sec. III, the FS of $\mathrm{BaFe}_{2} \mathrm{As}_{2}$ is similar to that of LaFeAsO but all cylinders, except the $\bar{\Gamma}$ centered $d_{x y}$-like hole cylinder, are far more warped in $k_{z}$ direction. Besides, the bct symmetry mixes $k_{z}$ dispersion into the $\left(k_{x}, k_{y}\right)$ dispersions. Although the nesting for $q=\overline{\mathrm{X}}$ is good when $k_{z}=\pi / 2 c$, it deteriorates at other $k_{z}$. Nevertheless, a peak of $\operatorname{Im} \chi_{0}(\mathbf{q})$, mostly due to the $d_{x y}$ states, is still present at $\overline{\mathrm{X}}$ (right-hand side of Fig. (4), and the behavior of $\operatorname{Re} \chi_{0}(\mathbf{q})$ for $y=0$ is qualitatively similar to that for LaFeAsO. This comparison leads to a conclusion that independently of the fine details of FS nesting, the formation of commensurate stripe order in the undoped compounds is preferable also in the limit of a weak magnetism.

Hole doping makes the hole sheets grow and the electron sheets shrink. The nesting with $\mathbf{q}=\overline{\mathrm{X}}$ is, nonethe- less, destroyed just as effectively as by electron doping. As a consequence, the real and imaginary parts of the susceptibility at $\overline{\mathrm{X}}$ are strongly suppressed. In contrast to $\mathrm{LaFeAsO}_{1-x} \mathrm{~F}_{x}$, the peak in $\operatorname{Re} \chi_{0}(\mathbf{q})$ shifts toward $\bar{\Gamma}$, like the energy minimum calculated for spin spirals in $\mathrm{Ba}_{1-2 y} \mathrm{~K}_{2 y} \mathrm{Fe}_{2} \mathrm{As}_{2}$ (Fig. (4) for the highest doping. The appearance of small- $q$, small-moment spin spirals correlates with the flat nonvanishing behavior of $\operatorname{Re} \chi_{0}(\mathbf{q})$ near $\bar{\Gamma}$.

\section{CONCLUSIONS}

In conclusion, our LSDA total-energy calculations for spin spirals in $\mathrm{LaFeAsO}_{1-x} \mathrm{~F}_{x}$ and $\mathrm{Ba}_{1-2 y} \mathrm{~K}_{2 y} \mathrm{Fe}_{2} \mathrm{As}_{2}$ using the experimental crystal structures confirm that in the undoped compounds the minimum of the total energy is reached at the wave vector $\mathbf{q}=\overline{\mathrm{X}}(1,0)$, which corresponds to stripe AFM order. The stability of this solution is, however, strongly affected by doping. With electron doping $(x)$ exceeding 0.1 in $\mathrm{LaFeAsO}_{1-x} \mathrm{~F}_{x}$, the minimum becomes shallow and shifts toward $\bar{M}(1,1)$ to an incommensurate wave vector. This destabilization of the commensurate collinear stripe order by electron doping is a band-structure effect but not sensitive to the details of Fermi surface nesting. Hole doping $(y)$ in $\mathrm{Ba}_{1-2 y} \mathrm{~K}_{2 y} \mathrm{Fe}_{2} \mathrm{As}_{2}$ roughly continues the trend calcu- 


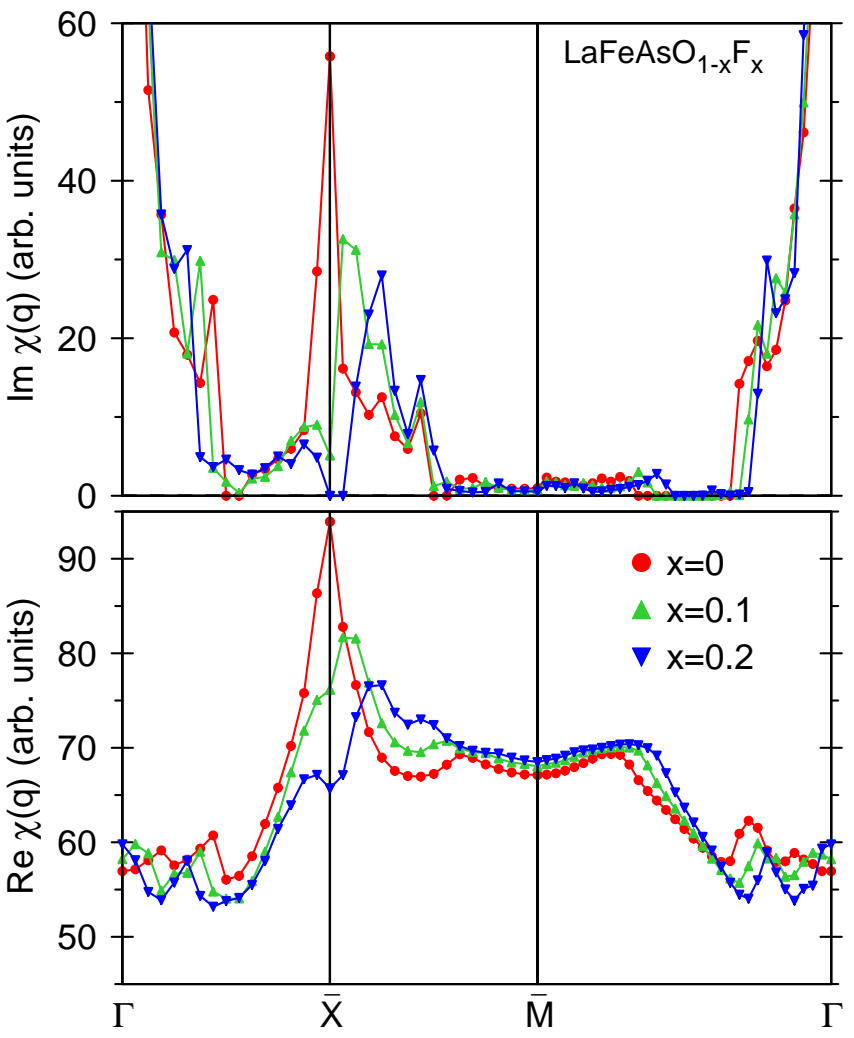

FIG. 8: (Color online) Imaginary and real parts of the bare susceptibility calculated for $\mathrm{LaFeAsO}_{1-x} \mathrm{~F}_{x}$ without shifting of the $d_{x y}$ energy. lated for decreasing electron doping in $\mathrm{LaFeAsO}_{1-x} \mathrm{~F}_{x}$, that is, $y \sim-x$. The energy gain due to stripe formation decreases with hole doping, but the minimum stays at $\overline{\mathrm{X}}$ for $y \lesssim 0.25$. In both compounds, the deviation of the $\mathbf{q}$ dependence of the energy from that of the classical Heisenberg model with nearest- and nextnearest-neighbor interactions becomes more pronounced with doping.

We found that even in the parent compounds the total energy varies strongly when two interpenetrating AFM sublattices formed by the Fe ions are rotated with respect to each other. The dependence of the energy on the angle between the Fe moments in the two sublattices cannot be described by the simple $j_{1}-j_{2}$ Heisenberg model, but may be reproduced by a biquadratic term proportional to $\left(\mathbf{S}_{i} \cdot \mathbf{S}_{j}\right)^{2}$, which favors collinear stripe AFM order.

Although the LSDA for the experimental crystal structures gives a stripe moments around $1.5 \mu_{B} / \mathrm{Fe}$ and concomitant eV-large exchange splittings of degenerate $\varepsilon_{j, \mathbf{k}}$ and $\varepsilon_{j^{\prime}, \mathbf{k}+\mathbf{q}}$ bands, linear-response calculations of the real and imaginary parts of the static noninteracting susceptibility based on the paramagnetic LDA band structure give similar results as the charge- and spin-self-consistent spin-spiral calculations.

Authors are grateful to G. Jackeli and L. Boeri for helpful discussions. V.N. Antonov gratefully acknowledges the hospitality at Max-Planck-Institut für Festkörperforschung in Stuttgart during his stay there. This work was partially supported by Science and Technology Center in Ukraine (STCU) inder Project No. 4930.
1 Y. Kamihara, T. Watanabe, M. Hirano, and H. Hosono, J. Am. Chem. Soc. 130, 3296 (2008).

2 Z. A. Ren, J. Yang, W. Lu, W. Yi, G. C. Che, X. L. Dong, L. L. Sun, and Z. X. Zhao, Materials Research Innovations 12, 105 (2008).

3 Z.-A. Ren, J. Yang, W. Lu, W. Yi, X.-L. Shen, Z.-C. Li, G.C. Che, X.-L. Dong, L.-L. Sun, F. Zhou, and Z.-X. Zhao, EPL 82, 57002 (2008).

${ }^{4}$ M. Rotter, M. Tegel, and D. Johrendt, Phys. Rev. Lett. 101, 107006 (2008).

${ }^{5}$ N. Ni, S. L. Bud'ko, A. Kreyssig, S. Nandi, G. E. Rustan, A. I. Goldman, S. Gupta, J. D. Corbett, A. Kracher, and P. C. Canfield, Phys. Rev. B 78, 014507 (2008).

6 M. Rotter, M. Tegel, D. Johrendt, I. Schellenberg, W. Hermes, and R. Poettgen, Phys. Rev. B 78, 020503(R) (2008).

7 T. Nomura, S. W. Kim, Y. Kamihara, M. Hirano, P. V. Sushko, K. Kato, M. Takata, A. L. Shluger, and H. Hosono, Supercon. Sci. Technol. 21, 125028 (2008).

8 T. Goko, A. A. Aczel, E. Baggio-Saitovitch, S. L. Bud'ko, P. C. Canfield, J. P. Carlo, G. F. Chen, P. Dai, A. C. Hamann, W. Z. Hu, H. Kageyama, G. M. Luke, J. L. Luo, B. Nachumi, N. Ni, D. Reznik, D. R. Sanchez-Candela, A. T. Savici, K. J. Sikes, N. L. Wang, C. R. Wiebe, T. J. Williams, T. Yamamoto, W. Yu, and Y. J. Uemura,
arXiv:0808.1425v1 (unpublished).

${ }^{9}$ Q. Huang, Y. Qiu, W. Bao, M. A. Green, J. W. Lynn, Y. C. Gasparovic, T. Wu, G. Wu, and X. H. Chen, Phys. Rev. Lett. 101, 257003 (2008).

10 C. de la Cruz, Q. Huang, J. W. Lynn, J. Li, I. Ratcliff, W., J. L. Zarestky, H. A. Mook, G. F. Chen, J. L. Luo, N. L. Wang, and P. Dai, Nature (London) 453, 899 (2008).

11 J. Dong, H. J. Zhang, G. Xu, Z. Li, G. Li, W. Z. Hu, D. Wu, G. F. Chen, X. Dai, J. L. Luo, Z. Fang, and N. L. Wang, EPL 83, 27006 (2008).

${ }^{12}$ H. Chen, Y. Ren, Y. Qiu, W. Bao, R. H. Liu, G. Wu, T. Wu, Y. L. Xie, X. F. Wang, Q. Huang, and X. H. Chen, EPL 85, 17006 (2009).

13 Z. P. Yin, S. Lebegue, M. J. Han, B. P. Neal, S. Y. Savrasov, and W. E. Pickett, Phys. Rev. Lett. 101, 047001 (2008).

14 T. Yildirim, Phys. Rev. Lett. 101, 057010 (2008).

15 S. Ishibashi, K. Terakura, and H. Hoson, J. Phys. Soc. Jpn. 77, 053709 (2008).

16 I. I. Mazin, D. J. Singh, M. D. Johannes, and M. H. Du, Phys. Rev. Lett. 101, 057003 (2008).

17 D. J. Singh and M.-H. Du, Phys. Rev. Lett. 100, 237003 (2008).

18 L. Boeri, O. V. Dolgov, and A. A. Golubov, Phys. Rev. 
Lett. 101, 026403 (2008).

19 D. J. Singh, Phys. Rev. B 78, 094511 (2008).

20 I. Opahle, H. C. Kandpal, Y. Zhang, C. Gros, and R. Valenti, Phys. Rev. B 79, 024509 (2009).

21 I. A. Nekrasov, Z. V. Pchelkina, and M. V. Sadovskii, JETP Lett. 88, 144 (2008).

22 I. I. Mazin, M. D. Johannes, L. Boeri, K. Koepernik, and D. J. Singh, Phys. Rev. B 78, 085104 (2008).

23 O. K. Andersen, Phys. Rev. B 12, 3060 (1975).

24 J. P. Perdew and Y. Wang, Phys. Rev. B 45, 13244 (1992).

25 V. N. Antonov, A. Y. Perlov, A. P. Shpak, and A. N. Yaresko, J. Magn. Magn. Mater 146, 205 (1995).

${ }^{26}$ O. K. Andersen and L. Boeri (unpublished).

27 P. Lee (unpublished).

28 A. I. Coldea, J. D. Fletcher, A. Carrington, J. G. Analytis, A. F. Bangura, J. H. Chu, A. S. Erickson, I. R. Fisher, N. E. Hussey, and R. D. McDonald, Phys. Rev. Lett. 101, 216402 (2008).
29 P. Blacha, K. Schwarz, G. K. H. Madsen, D. Kvasnica, and J. Luitz, WIEN2K, An augmented planewave+local orbitals program for calculating crystal properties (Technische Universität Wien, Austria) (2002), URL http://www.wien2k.at

30 O. Gunnarsson, J. Phys. F: Met. Phys. 6, 587 (1976).

31 U. K. Poulsen, J. Kollar, and O. K. Andersen, J. Phys. F: Met. Phys. 6, L241 (1976).

32 L. M. Sandratskii, J. Phys.: Condens. Matter 3, 8565 (1991).

33 S. Sharma, J. K. Dewhurst, S. Shallcross, C. Bersier, F. Cricchio, A. Sanna, S. Massidda, E. K. U. Gross, and L. Nordström, arXiv:0810.4278v2 (unpublished).

34 J. Callaway and C. Wang, J. Phys. F: Met. Phys. 5, 2119 (1975).

35 V. Yushankhai, A. Yaresko, P. Fulde, and P. Thalmeier, Phys. Rev. B 76, 085111 (2007). 